\title{
Creencias Epistemológicas en Profesores y su Relación con el Desarrollo Profesional desde la Evaluación Docente
}

\section{Teachers' Epistemological Beliefs and their Relationship with Professional Development Linked to the Teacher Evaluation System}

\author{
Sylvia Vargas, Marigen Narea y David Torres-Irribarra \\ Escuela de Psicología, Pontificia Universidad Católica de Chile
}

\begin{abstract}
El desarrollo profesional es fundamental para la calidad de la educación y los sistemas de evaluación docente pueden contribuir a su fortalecimiento. En este estudio se analizó la relación entre las creencias epistemológicas de los docentes y el desarrollo profesional a partir de la evaluación docente en una muestra no probabilística intencionada de profesores de 9 comunas de la Región del Maule, Chile. Los datos fueron recolectados a través del cuestionario de creencias epistemológicas y el cuestionario de consecuencias percibidas de la evaluación docente para el desarrollo profesional, ambos aplicados a 251 profesores de enseñanza básica de zonas urbanas y rurales; hombres y mujeres entre 25 y 66 años. Se realizaron análisis de correlación entre las variables, mediante $r$ de Spearman y un modelo de ecuaciones estructurales. Los resultados demuestran que docentes con creencias ingenuas sobre el conocimiento tienen una mayor percepción de que la evaluación docente contribuye a su desarrollo profesional. Se observan diferencias, dependiendo del contexto: profesores rurales perciben que favorece la autorreflexión sobre la práctica docente y profesores urbanos, que contribuye al aprendizaje, a través del trabajo colaborativo. Estos hallazgos evidencian que características personales de los profesores, como sus creencias y otros factores del contexto, influyen en las consecuencias de la evaluación docente para su desarrollo profesional. Se discute las limitaciones de contar con estándares uniformes en políticas educativas como la evaluación docente nacional, considerando la influencia de factores personales y contextuales en función de los resultados obtenidos.
\end{abstract}

Palabras clave: evaluación docente, creencias epistemológicas, desarrollo profesional, urbano, rural

Professional development is an essential factor in ensuring high-quality education and teacher evaluation systems can help to strengthen it. In this study, the relationship between teachers' epistemological beliefs and professional development associated with the teacher evaluation system is analyzed in a purposive, non-probabilistic sample of teachers from 9 municipalities in the Maule Region, Chile. The data were collected using the Epistemological Beliefs Questionnaire and the Questionnaire of Perceived Consequences of Teacher Evaluation Systems for Professional Development. Both instruments were administered to 251 primary education teachers from urban and rural areas; men and women between 25 and 66 years old. Correlation analyses were performed between the variables using Spearman's $r$ and a structural equation model. Results show that teachers with naive beliefs about knowledge are more likely to believe that teacher evaluation systems contribute to their professional development. Context-related differences are observed: rural teachers perceive that teacher evaluation processes foster self-reflection on teaching practice, while urban teachers consider that they enrich learning through collaborative work. These findings indicate that teachers' personal characteristics, such as their beliefs and other contextual factors, influence the impact of teacher evaluation systems on their professional development. The limitations of having uniform standards in educational policies such as the national teacher evaluation system are discussed, considering the evidence obtained regarding the influence of personal and contextual factors.

Keywords: teacher evaluation, epistemological beliefs, professional development, urban, rural

Existe consenso en que la calidad docente es un factor clave para el logro de los estudiantes (Accomplished California Teachers, 2015), por lo que promover y mantener la calidad del profesorado pasa a ser una prioridad política para alcanzar un alto rendimiento en todos los estudiantes (Looney, 2011).

Marigen Narea (i) https://orcid.org/0000-0001-7780-7425

El artículo es parte de la tesis de la primera autora para Optar al Grado de Magíster en Psicología Educacional de la Pontificia Universidad Católica de Chile.

La correspondencia relativa a este artículo debe ser dirigida a Sylvia Vargas, Escuela de Psicología, Pontificia Universidad Católica de Chile, Vicuña Mackenna 4860, Macul, Santiago, Región Metropolitana, Chile. Email: savargas1@uc.cl 
Un componente fundamental para la formación de habilidades y el logro de altos estándares de calidad docente, que impacten en el aprendizaje de los estudiantes, es el desarrollo profesional (Gore et al, 2017; Kyriakides et al., 2017; Reddy et al., 2015).

En la Encuesta Internacional de Enseñanza y Aprendizaje TALIS 2018 (Teaching and Learning International Survey) se indica que el desarrollo profesional debe ser visto en términos de aprendizaje permanente o continuo para la mejora de las prácticas docentes. También se informa que los profesores de países pertenecientes a la Organisation for Economic Co-operation and Development [OECD], en promedio, reportan que una de las estrategias de formación continua que ha tenido un mayor impacto en ellos ha sido aquella basada en la colaboración y los enfoques de colaboración para la docencia (OECD, 2019).

En la encuesta TALIS 2013 para Chile se advertía que un Plan Nacional Docente debía contemplar la reflexión profesional temprana; evaluaciones formativas y desarrollo profesional continuo que contemple las comunidades profesionales (Centro de Estudios MINEDUC, 2015). En la versión TALIS 2018 se señala que el $87 \%$ de los docentes chilenos participó en el último año de algún tipo de formación continua, el $57 \%$ a través de cursos y seminarios (OECD, 2019).

Como estrategia para fortalecer la profesión docente y contribuir a mejorar la calidad de la educación, Chile y otros países, como Estados Unidos, Inglaterra, Canadá, Portugal y Singapur, han implementado sistemas de evaluación docente (Santiago \& Benavides, 2009); no obstante, estos muestran diferentes características y énfasis (Flores, 2012). Por un lado, se considera que la evaluación docente debería ser formativa y promover el desarrollo profesional de los docentes y, por otro, que debería ser usada de manera sumativa para que los profesores den cuenta de su desempeño (Avalos \& Assael, 2006; Stronge, 2006). Algunos autores señalan la importancia de combinar ambos propósitos para mejorar la competencia docente (Stronge, 1995).

En Chile, el Sistema de Evaluación del Desempeño Profesional Docente manifiesta un propósito sumativo vinculado a la rendición de cuentas sobre el desempeño docente (Flotts \& Abarzúa, 2011), pero también declara como principal propósito la mejora de la calidad del profesorado (Aprueba reglamento sobre evaluación docente, 2004; Bonifaz, 2011), entregando retroalimentación que promueva una reflexión personal y oriente decisiones de desarrollo profesional (Flotts \& Abarzúa, 2011). Así, un sistema de evaluación docente tendría un importante rol en el desarrollo profesional, vinculado con su propósito formativo. Sin embargo, tal propósito, en comparación con su propósito sumativo, es aun débil y requiere ser fortalecido (Roa-Tampe, 2017; Taut et al., 2012).

La revisión de la literatura indica que factores contextuales, como el clima o la cultura escolar, las actitudes hacia la evaluación docente de líderes escolares (Taut et al., 2011) y otros factores propios del sistema de evaluación, como la claridad de sus propósitos y sus criterios de evaluación (Delvaux et al., 2013), influyen en los usos y efectos de la evaluación docente en términos de su propósito formativo. Sin embargo, también se han estudiado algunos factores individuales, como las creencias docentes respecto de los sistemas de evaluación y su vínculo con la motivación hacia su uso para el desarrollo profesional (Hyman, 2018; Reddy et al., 2018).

De acuerdo con Biesta et al. (2015), las creencias orientan hacia el futuro y, por lo tanto, pueden desempeñar un rol particular para conducir o motivar el logro de la agencia docente. Esta capacidad para influir intencionalmente en sus acciones es fundamental para el cambio (Bandura, 2008). Por esta razón, algunos estudios han vinculado las creencias docentes con el desarrollo profesional, encontrando que creencias de alto rango sobre la enseñanza y el aprendizaje se relacionan con mayor participación en actividades de desarrollo profesional (de Vries et al., 2014).

En particular, las creencias epistemológicas de los individuos influyen en sus formas de tratar y resolver problemas y en la toma de decisiones, razón por la que han sido vinculadas al desarrollo profesional (Geijsel et al., 2009; Harteis et al., 2006). Así, es fundamental conocer las creencias de los docentes para poder comprender y fortalecer su labor y la educación en general (Castro et al., 2015), lo cual también es un objetivo de los sistemas de evaluación docente.

Es relevante considerar, sin embargo, que la agencia docente se logra en entornos concretos y en condiciones ecológicas particulares (Biesta et al., 2015), por lo tanto, contar con antecedentes individuales permitirá establecer cursos de acción específicos desde políticas educativas diferenciadas, reconociendo que, como señalan Castillo-Miranda et al. (2017), la labor docente es heterogénea y, por ello, un sistema universal 
o estandarizado no puede alcanzar la riqueza de la diversidad de prácticas, necesidades y realidades de la profesión docente.

Este estudio tuvo el objetivo de contribuir a generar evidencia acerca de los factores a nivel de profesor que se relacionan con el desarrollo profesional a partir de la evaluación docente. La pregunta guía de esta investigación fue: ¿Cómo las creencias epistemológicas se relacionan con las consecuencias percibidas de la evaluación docente para el desarrollo profesional? Dada la evidencia sobre la importancia del contexto, también surgió la pregunta: ¿Existen diferencias en esta relación dependiendo del contexto urbano o rural?

\section{Evaluación Docente y Desarrollo Profesional}

Con el aumento de la demanda y el reconocimiento público sobre la importancia de la calidad docente, se ha comenzado a explorar y discutir la práctica de integrar el desarrollo profesional y la evaluación de los profesores (Smylie, 2014; Tuytens \& Devos, 2011).

Un sistema de evaluación docente impacta en el desarrollo profesional de los docentes, a través de una mejora en los conocimientos y prácticas de enseñanza de los profesores (Santiago \& Benavides, 2009). La evaluación docente repercute en una mayor reflexión sobre la práctica docente y más experimentación o aplicación de nuevas ideas de parte de los profesores, quienes también han indicado que a partir de la evaluación intentaron mejorar sus clases y su gestión en el aula (Heneman III \& Milanowski, 2003; Isoré, 2009). Su et al. (2017), así como Macfarlane y Gourlay (2009), plantean que, desde un modelo enfocado en el sistema de evaluación para el desarrollo profesional, los estándares profesionales de evaluación pueden apuntar a la autorregulación y reflexión en los profesores.

El desarrollo profesional tiene relación con oportunidades de aprendizaje formales e informales que profundizan y amplían las competencias profesionales de los docentes (Richter et al., 2011).

Las oportunidades de aprendizaje formales son definidas como ambientes de aprendizaje estructurados con un currículum específico (Feiman-Nemser, 2001) y vinculadas al modelo de perfeccionamiento (Little, 1993), lo que se conoce como la visión tradicional sobre el desarrollo profesional (Lieberman, 1995). En contraste, las oportunidades de aprendizaje informales no siguen un currículum específico, no se restringen a determinados ambientes (Desimone, 2009) y no son un mandato (Eurydice, 2008). Además, estas oportunidades a menudo tienen lugar en el contexto escolar, lo cual permite a los profesores reflexionar sobre su práctica y aprender de sus colegas (Putnam \& Borko, 2000).

Este estudio alude al desarrollo profesional docente en un contexto informal, puesto que el sistema de evaluación docente nacional se orienta principalmente, según la evidencia, al desarrollo profesional desde la reflexión que se genera en el proceso de elaboración de la evidencia solicitada (Roa-Tampe, 2017). Los planes de superación profesional, vinculados a un contexto formal de aprendizaje a partir de la evaluación docente, solo están destinados a docentes que no alcanzaron niveles mínimos de desempeño y cuyo porcentaje ronda el 20\% de los docentes, según datos del Centro de Perfeccionamiento, Experimentación e Investigaciones Pedagógicas [CPEIP] (2019).

En este estudio se entiende el uso y efecto formativo de la evaluación docente como un aprendizaje reflexivo, colaboración entre pares y un compromiso del profesor con su práctica. Esto es consecuente con lo señalado en un estudio de metodología cualitativa sobre el proceso de validación del Sistema de Evaluación del Desempeño Profesional Docente en Chile. En esta investigación Taut et al. (2012) reportan las consecuencias intencionadas percibidas por distintos grupos que participan de la evaluación. Como consecuencias se señalan efectos de la evaluación docente en la promoción del cambio de las prácticas docentes a partir de la autorreflexión y revisión de las propias prácticas, así como usos de la evaluación docente asociados a la promoción del trabajo entre pares, ya sea durante el proceso de evaluación o luego de este, a partir del análisis de resultados.

Aunque la evidencia señala que la evaluación docente puede proporcionar información y estimular el crecimiento profesional de los profesores (Isoré, 2009; Stronge, 2006), es una relación que históricamente no ha estado clara (Su et al., 2017). En efecto, no toda la literatura confirma la influencia positiva del sistema de evaluación docente en el desarrollo profesional, pues este no siempre es preciso y el proceso de evaluación puede ser insuficiente para mejorar el rendimiento docente (Isoré, 2009). Esta falta de impacto en el desarrollo profesional es a menudo una consecuencia de sistemas deficientes de evaluación, por ejemplo, falta de retroalimentación, ausencia de vínculo entre la evaluación y la práctica en el aula o incompatibilidad entre fines formativos y sumativos (Tucker, 1997). 
VARGAS, NAREA Y TORRES-IRRIBARRA

Su et al. (2017) advierten que, aunque los estándares explícitos son teóricamente necesarios para orientar diversas disciplinas y contextos docentes, se corre el riesgo de que el aprendizaje del profesor, en vez de comenzar por preocupaciones específicas vinculadas a su realidad, se convierta en una actividad técnicamente acumulativa y discursiva, desacoplada de los motivos éticos de los profesores y de la complejidad de la profesión.

\section{Factores que Influyen en las Consecuencias de la Evaluación Docente para el Desarrollo Profesional}

Existe cierta evidencia sobre factores culturales y organizacionales que podrían influir en las consecuencias de la evaluación docente para el desarrollo profesional, como lo son el liderazgo transformacional y el liderazgo instruccional (Runhaar et al., 2010; Tuytens \& Devos, 2011), así como la actitud positiva o negativa de un director hacia las evaluaciones de los profesores (Peterson \& Peterson, 2006). Asimismo, en un estudio en Chile, respecto de los usos y efectos percibidos de la evaluación, se concluye que mayores usos y efectos positivos asociados al propósito formativo se relacionarían con el clima escolar positivo y con las actitudes de los líderes hacia la evaluación docente, legitimando el sistema y participando activamente en el proceso (Taut et al., 2011).

Por otra parte, características propias del sistema de evaluación, como su equidad y la claridad de los criterios y propósitos de evaluación, influyen en las consecuencias para el desarrollo profesional (Delvaux et al., 2013).

Aunque escasos, hay estudios que han abordado factores individuales que influyen en las consecuencias para el desarrollo profesional. Los hallazgos señalan que la satisfacción de los profesores con su evaluación y sus entrevistas de desempeño influyen positivamente en el desarrollo docente (Delvaux et al., 2013). Asimismo, un estudio que investigó las percepciones de docentes hacia el sistema de evaluación en Texas encontró que profesores con creencias positivas hacia el uso de rúbricas, el trabajo colaborativo y la reflexión generada a partir de la evaluación docente tenían una mayor disposición a hacer uso de ella para mejorar sus prácticas (Hyman, 2018). Por otra parte, un estudio que midió las actitudes y creencias de docentes respecto de la evaluación docente en Nueva Jersey evidencia que los profesores valoran altamente la comunicación colaborativa con respecto al proceso de evaluación, así como los comentarios de evaluación constructivos, claros y específicos. Aun así, el sistema de evaluación no motivó a los maestros a cambiar después de la evaluación, teniendo un impacto mínimo en su desarrollo profesional (Reddy et al, 2018).

Estas diferencias en la relación entre creencias y desarrollo profesional invitan a considerar que, como señala Schommer (1990), las creencias son sistemas dentro de otros sistemas y, por lo tanto, están fuertemente impregnadas por la cultura (Hofer \& Pintrich, 1997). En efecto, la agencia docente, es decir, la motivación del uso de la evaluación para el cambio, también dependerá de factores estructurales, culturales, organizacionales e incluso materiales para favorecer su desarrollo (Biesta et al., 2015).

El desarrollo profesional también puede verse afectado por dos contextos distintos, como el urbano y rural. La dispersión geográfica de la escuela rural tiende a afectar a los docentes que se encuentran en condiciones poco favorables para compartir experiencias y reflexiones pedagógicas (Ministerio de Educación [MINEDUC], s.f.). Por otra parte, la ambigüedad de rol que perciben los docentes rurales chilenos, por tener que desempeñar múltiples tareas, podría afectar negativamente la competencia profesional (Rubio González et al., 2019). En un estudio en Indonesia, cuyo fin era probar la efectividad de un programa de desarrollo profesional, se evidenció que los profesores de escuelas rurales estaban en desventaja respecto de la efectividad del programa, frente a los urbanos, por condiciones como el número de estudiantes, problemas de disciplina y recursos inadecuados, lo que complejizaba su implementación (Soebari \& Aldridge, 2016).

Considerando, entonces, que el desarrollo profesional sería un resultado deseado de la evaluación docente, pero que algunos estudios indican que es insuficiente su impacto positivo en el desarrollo profesional (Isoré, 2009), surge la necesidad de estudiar posibles factores que pudieran influir en la elección del profesor para participar o no en el desarrollo profesional, aún más cuando es escasa la literatura referida a estas influencias a nivel de profesor. 


\section{Evaluación Docente en Chile}

El Sistema de Evaluación del Desempeño Profesional Docente Nacional es parte de la consolidación de las políticas educacionales orientadas al fortalecimiento de la profesión docente y de la calidad de la educación desde inicios de los años 90. Fue introducida por ley e impulsada por el Ministerio de Educación en el año 2003, a raíz de un acuerdo entre este organismo, la Asociación Chilena de Municipalidades y el Colegio de Profesores de Chile (Bonifaz, 2011).

La participación de los profesores en la evaluación es obligatoria desde el año 2005. Desde su implementación hasta el año 2019, se han realizado más de 245.000 evaluaciones correspondientes a educación parvularia, enseñanza básica, educación media, educación especial y educación de adultos (DocenteMás, s.f.a).

Desde el año 2016, con la incorporación de la ley que crea el Sistema de Reconocimiento del Desarrollo Profesional Docente, más conocido como la Carrera Docente, los profesores pueden demostrar las competencias alcanzadas y la experiencia en el ejercicio, siendo categorizados en determinado tramo de desarrollo (inicial, acceso, temprano, avanzado, experto), de acuerdo con sus años de experiencia y resultado vigente en el instrumento Portafolio de la Evaluación Docente. Así, la evaluación docente adquiere gran relevancia, ya que los resultados obtenidos por los profesores pasan a ser uno de los factores que definen su tramo de desarrollo. Además, el avance de un tramo a otro significará la posibilidad de asumir nuevas responsabilidades y obtener mayores remuneraciones (MINEDUC, 2016). Asimismo, desde el año 2019, su rendición es voluntaria para docentes que se encuentran en los tramos Experto I y II de la Carrera Docente (CPEIP, 2019).

El modelo chileno de evaluación docente, aunque recoge un propósito sumativo y formativo, se fundamenta principalmente en las prácticas docentes basadas en estándares profesionales. De esta manera, se siguen las tendencias internacionales en términos de evaluación docente, las que muestran un creciente interés hacia los estándares acerca del desempeño (Isoré, 2009), con el fin de entregar retroalimentación precisa que promueva una reflexión personal y oriente decisiones de desarrollo profesional para mejorar la práctica docente (Flotts \& Abarzúa, 2011).

Para obtener información pertinente tanto al propósito sumativo como formativo y analizar con mayor riqueza y profundidad el desempeño, el sistema nacional docente ha recurrido a instrumentos de medición, como un portafolio (referido al diseño, implementación y evaluación de una unidad pedagógica y una clase filmada), la entrevista por un evaluador par, el informe de referencia de terceros (director del establecimiento educacional y del jefe de la Unidad Técnico Pedagógica) y una autoevaluación.

La evaluación final de cada profesor corresponde a una apreciación global relativa al desempeño profesional relacionado con los dominios y criterios del Marco para la Buena Enseñanza, el que contiene los estándares que definen una adecuada práctica profesional docente y el conjunto de responsabilidades que debe asumir un profesor (CPEIP, 2008). El Marco constituye el fundamento para evaluar el desempeño de los profesores, estableciendo cuatro niveles de desempeño (DocenteMás, s.f.b):

- Destacado: Desempeño profesional que clara y consistentemente sobresale con respecto a lo que se espera en el indicador evaluado. Manifiesta un amplio repertorio de conductas respecto a lo que se está evaluando, o bien, riqueza pedagógica que se agrega al cumplimiento del indicador.

- Competente: Desempeño profesional adecuado. Cumple con lo requerido para ejercer profesionalmente el rol docente. No es excepcional, pero se trata de un buen desempeño.

- Básico: Desempeño profesional que cumple con lo esperado en el indicador evaluado, pero con cierta irregularidad (ocasionalmente).

- Insatisfactorio: Desempeño que presenta claras debilidades en el indicador evaluado y estas afectan significativamente el quehacer docente.

Los resultados obtenidos son reportados a cada profesor evaluado, indicando su categoría general y entregando el detalle de sus resultados para cada una de las dimensiones evaluadas. También se entrega un informe a la escuela y al municipio.

Para docentes con resultados bajo el estándar, especialmente aquellos calificados en el nivel de desempeño más bajo (insatisfactorio), la evaluación docente chilena establece altas consecuencias negativas, ya que pueden abandonar la dotación docente si no logran mejorar sus resultados al año siguiente. Como señala Cox (2007), pareciera que las políticas educativas marcharan hacia la presión y el control, generando 
un desequilibrio entre exigencia y apoyo, alejándose de los propósitos formativos. Cabezas et al. (2019) advierten que, si los profesores no pueden reconocer el valor formativo de los sistemas de evaluación docente, el propósito sumativo y sus consecuencias económicas (vinculadas a la carrera docente) podrían distorsionar el rol formativo de la evaluación para el desarrollo profesional.

\section{Creencias Epistemológicas en Docentes}

La influencia de las creencias docentes en la implementación del cambio y la innovación ha sido reconocida como un aspecto importante, afirmándose que estas pueden llegar a determinar si un profesor se inclina a un cambio particular o no (Chan \& Elliott, 2002; Fullan \& Stiegelbauer, 1997), por lo que las creencias podrían tener un impacto en la forma en que los docentes se enfrentan al desarrollo profesional.

Richardson (1996) señala que las creencias aluden a entendimientos psicológicos, premisas o proposiciones sobre el mundo que se cree que son verdaderas. Según este mismo autor, las creencias asociadas con el desarrollo profesional del docente implican las comprensiones generales relacionadas con el aprendizaje que un profesor sostiene que son ciertas.

A través de las creencias, los profesores perciben, organizan y procesan la información y toman las decisiones respecto de su trabajo profesional, puesto que constituyen un potente factor en la determinación de sus conductas (Fang, 1996).

Algunas creencias frecuentemente relacionadas con la investigación educacional aluden a las creencias epistemológicas. Las creencias epistemológicas corresponden a enunciados personales fundamentales acerca de la naturaleza del conocimiento y del aprendizaje (Hofer \& Pintrich, 1997). Para Schommer (1990), el sistema de creencias epistemológicas se compone de cuatro dimensiones, cada una de las cuales se tipifica, de acuerdo con su grado de desarrollo y complejidad, desde un polo de ingenuidad a un polo de sofisticación. Estas dimensiones se componen de creencias sobre la estructura del conocimiento, que iría desde que el conocimiento está formado por piezas desordenadas a que el conocimiento es integrado; creencias sobre la estabilidad del conocimiento, que iría desde que el conocimiento es tentativo a que el conocimiento es inmodificable y certero; creencias sobre el control del aprendizaje, que iría desde que la capacidad para aprender está determinada desde el nacimiento a la creencia en que esta capacidad se puede ir incrementando durante la vida; y la creencia respecto a la velocidad del aprendizaje, que iría desde que el aprendizaje ocurre rápido, o no ocurre, a la creencia en que es un proceso gradual.

Como teorías subjetivas de un individuo, las creencias epistemológicas tienen la función de dirigir y controlar las acciones (Paechter et al., 2013). Estas creencias se desempeñarían como filtros que determinan el desarrollo de ciertas estrategias motivacionales que impactan en procesos de aprendizaje autorregulado (Hofer \& Pintrich, 1997).

Un sistema de creencias elaborado se relaciona con un mejor desempeño en el proceso de aprendizaje (Hofer, 2004). También hay evidencia que indican resultados negativos en el proceso de aprendizaje, si este es guiado por una visión epistemológica naturalista o ingenua (Harteis et al., 2006).

Son escasas las investigaciones que se han realizado sobre las creencias epistemológicas de los docentes (Guerra Zamora \& Sebastián Balmaceda, 2015; Hofer, 2004) y más escasas aún en relación con el desarrollo profesional (de Vries et al., 2014; Harteis et al., 2006). No obstante, Guerra Zamora y Sebastián Balmaceda (2015) señalan que las creencias epistemológicas de los docentes se relacionarían con sus procesos de aprendizaje, los que se verían afectados negativamente por creencias ingenuas respecto del conocimiento y el aprendizaje.

Harteis et al. (2006) señalan que el aprendizaje a nivel profesional análogamente está relacionado con el grado de elaboración de las creencias epistemológicas. Considerando el carácter reflexivo atribuido a la profesión docente y la formación continua exigida para un buen ejercicio profesional, se supone que los docentes se encuentran permanentemente en proceso de aprender. El grado de desarrollo de sus creencias epistemológicas podría establecer diferencias en este aspecto, considerando, además, que las investigaciones muestran asociaciones entre pensamiento de alto nivel y operar con creencias epistemológicas sofisticadas (Nussbaum \& Bendixen, 2003; Schommer-Aikins \& Hutter, 2002).

Resulta evidente que las creencias epistemológicas pueden influenciar el desarrollo profesional, ya que pueden restringir la percepción de los docentes respecto de sus posibilidades de acción. 
En un estudio a nivel nacional realizado en Inglaterra, cuyo objetivo era conocer el rol de las creencias, prácticas y experiencias sobre el aprendizaje en el desarrollo profesional de los docentes y en el cambio en su aprendizaje, se encontró que las creencias sobre el aprendizaje tienen una influencia moderada sobre qué y cómo aprenden los docentes, concluyendo que, más allá de las influencias individuales, se debe reconocer que el aprendizaje de los maestros también está influenciado por las condiciones de la organización (Opfer et al., 2011).

Un estudio cualitativo, a través de entrevistas semiestructuradas, con profesores universitarios alemanes buscaba conocer el impacto de las creencias epistemológicas en la percepción e interpretación de sus dificultades asociadas al aprendizaje. En tal estudio, los investigadores encontraron que la mayoría de los profesores tenía una visión naturalista del conocimiento y una percepción rígida del rol del profesor, lo que se relacionaba con su sistema de creencias epistemológicas menos elaboradas. Lo anterior llevaba a que los docentes percibieran las restricciones profesionales como permanentes y percibieran opciones limitadas en cuanto a su desarrollo, restringiendo la búsqueda de ayuda ante un problema. Finalmente concluyen que los profesionales con sistemas de creencias más elaboradas tenían una mayor comprensión de su trabajo como una fuente de aprendizaje y desarrollo profesional (Harteis et al., 2006).

Existe evidencia que señala que algunas variables personales se relacionan con la motivación hacia el desarrollo profesional en docentes, a partir de la evaluación docente. En un estudio sobre las actitudes y creencias de los docentes hacia el sistema de evaluación docente se indica que aquellos profesores que recibieron un beneficio directo a partir de los resultados de la evaluación docente tendieron a percibirla como menos amenazante y a correlacionar positivamente con la motivación hacia el cambio de sus prácticas. Por otra parte, aquellos docentes con más años de experiencia evidenciaron una menor motivación para orientarse hacia el cambio a partir de la evaluación docente. Sin embargo, no se encontraron hallazgos significativos respecto del grado educativo (Reddy et al., 2018).

Dada la evidencia descrita, este estudio esperaba encontrar que aquellos docentes que reportaran creencias epistemológicas más sofisticadas manifestaran mayores consecuencias de la evaluación docente para su desarrollo profesional. Asimismo, se esperaba encontrar diferencias en la relación entre creencias epistemológicas y desarrollo profesional a partir de la evaluación docente, según ubicación geográfica y algunas variables personales, como años de experiencia, grado educacional y resultados de la evaluación docente.

\section{Método}

\section{Participantes}

De una población de 6.161 profesores de enseñanza básica que se desempeñan en 2017 en escuelas municipales de la región del Maule (Centro de Estudios MINEDUC, 2018), participaron 251 profesores de $1^{\circ}$ a $8^{\circ}$ año de enseñanza básica de 38 escuelas municipales de nueve comunas de la región del Maule. La muestra fue de tipo no probabilística y comprendió un muestreo intencionado, ya que se seleccionaron de la población aquellos profesores que habían sido evaluados por el Sistema de Evaluación Docente Nacional, lo cual responde a los propósitos del estudio. Los profesores que participaron requerían ser docentes de la región del Maule debido a la cercanía geográfica, respondiendo a un diseño muestral por conveniencia; pertenecer a una administración municipal ya que la evaluación docente iba dirigida a aquellos profesores; haber participado de al menos un proceso de evaluación docente.

Participaron 12 escuelas urbanas con una matrícula promedio de 414 estudiantes $(D E=212,5)$, con una media de 24 estudiantes por aula $(D E=5,4)$ y un promedio de 36 docentes $(D E=11,8)$. Del área rural, participaron 26 escuelas con una matrícula promedio de 139 alumnos $(D E=85,1)$, con una media de 13 estudiantes por curso $(D E=6,7)$ y un promedio de 18 docentes $(D E=7,2)$.

El $41 \%$ de los profesores participantes se desempeñaba en escuelas urbanas y el 59\%, en establecimientos rurales, lo que coincide con la situación de la región del Maule, puesto que el 31\% de sus establecimientos municipales pertenece al contexto urbano y el 69\%, al rural (Centro de Estudios MINEDUC, 2019). En la Tabla 1 se muestran las características sociodemográficas de los participantes.

Los criterios de inclusión para los participantes fueron ser profesor de la región del Maule, desempeñarse en una escuela básica de administración municipal y haber sido evaluado por el Sistema de Evaluación del Desempeño Profesional Docente. 
Tabla 1

Características Sociodemográficas de los Participantes

\begin{tabular}{|c|c|c|c|}
\hline \multirow{2}{*}{ Variable } & \multirow{2}{*}{ Categoría } & \multicolumn{2}{|c|}{ Total } \\
\hline & & Frecuencia & Porcentaje \\
\hline \multirow[t]{4}{*}{ Edad } & 25 a 34 años & 81 & 32,3 \\
\hline & 35 a 44 años & 71 & 29,1 \\
\hline & 45 a 55 años & 63 & 25,1 \\
\hline & 56 a 66 años & 34 & 13,5 \\
\hline \multirow[t]{2}{*}{ Género } & Masculino & 70 & 27,9 \\
\hline & Femenino & 181 & 71,1 \\
\hline \multirow[t]{5}{*}{ Nivel de estudios } & Pregrado & 101 & 40,2 \\
\hline & Diplomado & 6 & 2,4 \\
\hline & Postítulo & 112 & 44,6 \\
\hline & Magíster & 31 & 12,4 \\
\hline & Doctorado & 1 & 0,4 \\
\hline \multirow[t]{5}{*}{ Años de experiencia docente } & 1 a 10 años & 135 & 53,8 \\
\hline & 11 a 20 años & 60 & 23,9 \\
\hline & 21 a 30 años & 25 & 10,0 \\
\hline & 31 a 40 años & 28 & 11,2 \\
\hline & 41 a 50 años & 3 & 1,2 \\
\hline \multirow[t]{4}{*}{ Resultados obtenidos primera evaluación } & Insatisfactorio & 4 & 1,6 \\
\hline & Básico & 17 & 6,8 \\
\hline & Competente & 162 & 64,5 \\
\hline & Destacado & 68 & 27,1 \\
\hline \multirow[t]{3}{*}{ Resultados obtenidos segunda evaluación } & Básico & 7 & 4,8 \\
\hline & Competente & 100 & 69,0 \\
\hline & Destacado & 37 & 25,5 \\
\hline
\end{tabular}

El estudio se desarrolló con profesores de enseñanza básica, ya que una variable de interés era la comparación urbana versus rural. En el área rural de la región del Maule hay solo seis establecimientos con enseñanza media, de un total de 372 establecimientos rurales municipales (Centro de Estudios MINEDUC, 2019).

\section{Instrumentos}

\section{Cuestionario sobre Consecuencias Percibidas de la Evaluación Docente}

Para medir las consecuencias percibidas de la evaluación docente para el desarrollo profesional se utilizó un cuestionario autoadministrado de elaboración propia, ya que no existía un instrumento que evaluara esta variable. Su versión inicial constaba de 20 ítems que, luego del pilotaje, derivó en un cuestionario final de 18 ítems de respuesta cerrada con escala Likert de 4 puntos, donde 1 indica menor percepción de consecuencias para el desarrollo profesional y 4 mayor percepción de consecuencias para el desarrollo profesional.

Este instrumento se construyó en base a los hallazgos de investigaciones previas referidas a las consecuencias percibidas de la evaluación docente por líderes escolares (Taut et al., 2011), en base a los hallazgos respecto de la teoría subyacente de la evaluación docente (Taut et al., 2010) y a la teoría sobre aprendizaje informal (Desimone, 2009). Tal información permitió determinar las dos dimensiones que componen la variable: Autorreflexión (la evaluación docente ha favorecido la autoobservación de mis prácticas docentes) y Trabajo Colaborativo (la evaluación docente me ha permitido aprender de otros profesores con mejor desempeño). Esto se complementó con evidencia referida al contenido, a través de la revisión de expertos y de entrevistas cognitivas a cinco profesores.

$\mathrm{Al}$ realizar análisis factoriales confirmatorios con la muestra final, se corroboraron las dos dimensiones, alcanzando una varianza total explicada de $65,2 \%$ de las consecuencias percibidas. En cuanto a la consistencia interna, se obtuvo un alfa de Cronbach de 0,94 para la dimensión Autorreflexión y 0,93 para Trabajo Colaborativo. 


\section{Epistemological Beliefs Inventory (Bendixen et al., 1998; Schraw et al., 2002)}

Para la medición de las creencias epistemológicas se utilizó un cuestionario autoadministrado elaborado por el área de Medición y Estudio del Programa de Asignación de Excelencia Pedagógica (AEP) ${ }^{1}$, en base al Epistemological Beliefs Inventory (Bendixen et al., 1998; Schraw et al., 2002). Este cuestionario contempla cuatro escalas, que se corresponden con las cuatro dimensiones del constructo: Estructura del Conocimiento (los profesores debieran enseñar más hechos que ideas abstractas), Estabilidad del Conocimiento (lo que se enseña en los colegios es cierto hoy y será cierto mañana), Control del Aprendizaje (la gente inteligente nace asî) y Velocidad del Aprendizaje (la mayoría de las cosas que se aprenden, se aprenden a la primera). Cada dimensión tiene 6 ítems, sumando un total de 24 afirmaciones que deben ser evaluadas en una escala de 1 a 5, donde 1 representa las creencias más sofisticadas y 5, las creencias más ingenuas. Para este estudio se recodificaron los ítems, por lo que 1 representa creencias más ingenuas y 5, creencias más sofisticadas.

Aplicaciones anteriores entregan evidencia sobre la estructura interna, a través de análisis factoriales confirmatorios, identificando las cuatro dimensiones señaladas (Araya, 2005). Araya (2005) informa un índice de alfa de Cronbach de 0,77 y 0,74 para Estructura del Conocimiento y Estabilidad del Conocimiento, respectivamente, y un índice de 0,84 y 0,81 para Control del Aprendizaje y Velocidad del Aprendizaje, respectivamente. García y Sebastián (2011) dan cuenta de índices de alfa de Cronbach moderados: 0,63 y 0,70 para Estructura del Conocimiento y Estabilidad del Conocimiento, respectivamente, y 0,81 y 0,72 para Control del Aprendizaje y Velocidad del Aprendizaje, respectivamente. La consistencia interna de las escalas está por sobre los valores obtenidos por otros cuestionarios desarrollados para medir este tipo de creencias y es suficientemente alta para su uso en contextos de investigación.

\section{Procedimiento}

Luego de que el estudio fuera aprobado por el Comité de Ética y Seguridad en Investigación de la Pontifica Universidad Católica de Chile, se contactó a cuatro directores de escuelas municipales de la comuna de San Clemente de la región del Maule (dos rurales y dos urbanas), quienes accedieron a la realización del pilotaje del instrumento Consecuencias de la Evaluación Docente para el Desarrollo Profesional. Se aplicó a 36 profesores de enseñanza básica, luego de haber firmado el consentimiento informado.

Para la recolección final de datos se contactó a los directores de las escuelas, a quienes se les entregó información respecto de los objetivos y la recolección de información. Al momento de contar con la autorización, se acordaba también la forma de aplicación de los instrumentos.

En 18 escuelas se autorizó que el equipo investigador aplicara los instrumentos a los docentes durante consejo de profesores, instancia donde se dieron a conocer los objetivos y propósitos del estudio y se resguardaron aspectos éticos, a través de la firma de consentimiento informado, destacando la confidencialidad de las respuestas y, por lo tanto, el anonimato de los cuestionarios. La aplicación tuvo una duración promedio de 25 minutos. En las restantes 20 escuelas los equipos directivos gestionaron la aplicación, fijando un plazo de tiempo, luego del cual se retiraban los cuestionarios y consentimientos firmados de los establecimientos. En estos casos se dejaba un instructivo a los directivos, en el que se señalaban los criterios muestrales para su aplicación y los resguardos éticos, junto con la entrega de consentimientos informados.

El pilotaje del instrumento se desarrolló en diciembre de 2016. El proceso de aplicación de instrumentos se inició en mayo de 2017 y finalizó en agosto del mismo año.

\section{Análisis de Datos}

Las respuestas al cuestionario piloteado sobre Consecuencias Percibidas de la Evaluación Docente fueron analizadas mediante SPSS versión 23.

Para evaluar la estructura de dicho cuestionario se realizaron análisis factoriales confirmatorios generalizados (modelando las respuestas en formato Likert como ordinales), estimados usando el método WLSMV (sigla en inglés del método de estimación de mínimos cuadrados con pesos que incluye medias y varianzas ajustadas) en el programa Mplus 8.4. La consistencia interna se calculó usando el coeficiente alfa de Cronbach.

${ }^{1}$ Previa autorización de la Directora del Área de Desarrollo de Pruebas del Centro de Medición MIDE UC de la Escuela de Psicología de la Pontificia Universidad Católica de Chile. 
Para el estudio final se realizó un análisis del proceso de digitación, realizando una validación muestral con el fin de evitar errores de este tipo. Luego se llevó a cabo un análisis exploratorio, mediante un diagrama de caja y bigotes, para determinar la presencia de casos muestrales con comportamientos atípicos, lo que concluyó en la eliminación de dos casos. Por último, se realizaron análisis de correlación entre las variables a través de $r$ de Spearman, ya que este no tiene como supuesto la normalidad de los datos analizados.

Finalmente, y con el fin de corroborar los resultados estimados calculando correlaciones $r$ de Spearman, se estimó un modelo de ecuaciones estructurales generalizado para integrar de forma simultánea los distintos factores que pueden impactar sobre los atributos de autorreflexión y trabajo colaborativo. Se usó Mplus 8.4, con el que se estimó un modelo de regresión múltiple para (a) controlar simultáneamente los efectos de los distintos factores, (b) controlar el anidamiento de los profesores en colegios y (c) incluir en el mismo modelo los análisis factoriales confirmatorios generalizado de los atributos de autorreflexión, trabajo colaborativo y estructura y estabilidad del conocimiento.

\section{Resultados}

Como se muestra en la Tabla 2, los análisis indican la presencia de relaciones inversas débiles entre las dimensiones Estructura del Conocimiento y Estabilidad del Conocimiento con Autorreflexión y entre Estabilidad del Conocimiento y Trabajo Colaborativo. Así, en la medida en que disminuye el acuerdo con la creencia de que el conocimiento es integrado y tentativo, aumenta la percepción de que la evaluación docente favorece el desarrollo profesional, a través de la autorreflexión o el trabajo colaborativo.

Tabla 2

Correlaciones entre Dimensiones de las Creencias Epistemológicas y Dimensiones de las Consecuencias Percibidas de la Evaluación Docente

\begin{tabular}{lcc}
\hline & Autorreflexión & Trabajo colaborativo \\
\hline Estructura del conocimiento & $-0,151$ & $-0,040$ \\
& $(0,017)$ & $(0,529)$ \\
Estabilidad del conocimiento & $-0,140$ & $-0,237$ \\
& $(0,026)$ & $(<0,001)$ \\
Control del aprendizaje & 0,32 & $-0,055$ \\
& $(0,68)$ & $(0,383)$ \\
Velocidad del aprendizaje & 0,100 & 0,002 \\
& $(0,014)$ & $(0,081)$ \\
\hline
\end{tabular}

Nota. Entre paréntesis aparece $p$.

Respecto de las dimensiones Estructura del Conocimiento y Trabajo Colaborativo, se encuentran relaciones inversas solo a nivel de indicadores. Para las dimensiones Control del Aprendizaje y Velocidad del Aprendizaje con Autorreflexión, se encuentran relaciones directas solo a nivel de indicadores. No se encontraron relaciones a nivel de dimensión ni de indicadores entre las dimensiones Control del Aprendizaje y Velocidad del Aprendizaje con Trabajo Colaborativo.

Los análisis correlacionales según algunos niveles de las variables sociodemográficas indican relaciones inversas entre algunas dimensiones, como se muestra en la Tabla 3.

Para el nivel de estudio de postítulo, se encuentran relaciones inversas débiles para las dimensiones Estructura del Conocimiento y Estabilidad del Conocimiento y Autorreflexión y entre las dimensiones Estabilidad del Conocimiento y Trabajo Colaborativo. Esto implica que para los docentes con postítulo, en la medida en que creen que el conocimiento está conformado por partes y es certero, aumenta la percepción de que la evaluación docente favorece el desarrollo profesional, a partir de la autorreflexión y el trabajo colaborativo, o, al contrario, a medida que mantienen creencias más sofisticadas declaran un menor efecto y uso de la evaluación docente para su desarrollo. 
Tabla 3

Correlaciones entre Dimensiones de las Creencias Epistemológicas y Dimensiones de las Consecuencias Percibidas de la Evaluación Docente, según Niveles de Variables Sociodemográficas

\begin{tabular}{|c|c|c|c|}
\hline Variable y nivel & Dimensión & Autorreflexión & Trabajo colaborativo \\
\hline \multirow{8}{*}{$\begin{array}{l}\text { Nivel de estudios: } \\
\text { Postítulo }(n=112)\end{array}$} & \multirow[t]{2}{*}{ Estructura del conocimiento } & $-0,240$ & $-0,058$ \\
\hline & & $(0,011)$ & $(0,541)$ \\
\hline & \multirow[t]{2}{*}{ Estabilidad del conocimiento } & $-0,194$ & $-0,352$ \\
\hline & & $(0,041)$ & $(<0,001)$ \\
\hline & \multirow[t]{2}{*}{ Control del aprendizaje } & $-0,002$ & $-0,056$ \\
\hline & & $(0,984)$ & $(0,557)$ \\
\hline & \multirow[t]{2}{*}{ Velocidad del aprendizaje } & 0,064 & $-0,006$ \\
\hline & & $(0,501)$ & $(0,946)$ \\
\hline \multirow{7}{*}{$\begin{array}{l}\text { Años de experiencia: } \\
1 \text { a } 10 \text { años de experiencia }(n=135)\end{array}$} & \multirow[t]{2}{*}{ Estructura del conocimiento } & $-0,200$ & $-0,043$ \\
\hline & & $(0,020)$ & $\begin{array}{l}(0,619) \\
-0,09\end{array}$ \\
\hline & Estabilidad del conocimiento & $\begin{array}{r}-0,100 \\
(0,063)\end{array}$ & $(0,015)$ \\
\hline & \multirow[t]{2}{*}{ Control del aprendizaje } & 0,038 & $-0,129$ \\
\hline & & $(0,658)$ & $(0,136)$ \\
\hline & \multirow[t]{2}{*}{ Velocidad del aprendizaje } & 0,011 & $-0,069$ \\
\hline & & $(0,898)$ & $(0,423)$ \\
\hline \multicolumn{4}{|l|}{ Evaluación docente: } \\
\hline \multirow{7}{*}{$\begin{array}{l}\text { Competente } 1^{\mathrm{a}} \text { evaluación docente } \\
(n=162)\end{array}$} & Estructura del conocimiento & $-0,104$ & $-0,024$ \\
\hline & \multirow[t]{2}{*}{ Estabilidad del conocimiento } & $-0,182$ & $-0,280$ \\
\hline & & $(0,021)$ & $(<0,001)$ \\
\hline & \multirow[t]{2}{*}{ Control del aprendizaje } & $-0,040$ & $-0,068$ \\
\hline & & $(0,613)$ & $(0,388)$ \\
\hline & \multirow[t]{2}{*}{ Velocidad del aprendizaje } & 0,029 & 0,007 \\
\hline & & $(0,711)$ & $(0,932)$ \\
\hline \multirow{7}{*}{$\begin{array}{l}\text { Competente } 2^{\mathrm{a}} \text { evaluación docente } \\
(n=100)\end{array}$} & Estructura del conocimiento & $\begin{array}{c}-0,152 \\
(0,131)\end{array}$ & $\begin{array}{c}-0,049 \\
(0,630)\end{array}$ \\
\hline & \multirow[t]{2}{*}{ Estabilidad del conocimiento } & $-0,088$ & $-0,266$ \\
\hline & & $(0,385)$ & $(0,007)$ \\
\hline & \multirow[t]{2}{*}{ Control del aprendizaje } & 0,171 & 0,143 \\
\hline & & $(0,090)$ & $(0,156)$ \\
\hline & \multirow[t]{2}{*}{ Velocidad del aprendizaje } & 0,193 & 0,148 \\
\hline & & $(0,054)$ & $(0,143)$ \\
\hline
\end{tabular}

Nota. Entre paréntesis aparece $p$.

De acuerdo con los años de experiencia, se encuentran relaciones inversas débiles entre las dimensiones Estructura del Conocimiento y Autorreflexión y entre las dimensiones Estabilidad del Conocimiento y Trabajo Colaborativo en los profesores que tienen entre 1 y 10 años de experiencia. Los resultados indican que aquellos docentes que cuentan con menos experiencia, en la medida en que creen que el conocimiento es certero y formado por partes manifiestan mayor percepción de que la evaluación docente favorece su desarrollo profesional, a partir de la autorreflexión o el trabajo colaborativo.

De acuerdo con los resultados obtenidos en la primera evaluación docente, se encuentran relaciones inversas muy débiles entre las dimensiones Estabilidad del Conocimiento y Autorreflexión en los profesores que obtienen un desempeño competente. En aquellos docentes que obtienen un resultado competente en la primera y segunda evaluación, se encuentran relaciones inversas entre las dimensiones Estabilidad del Conocimiento y Trabajo Colaborativo. Lo anterior indica que aquellos docentes que obtienen mejor resultado en la evaluación docente, en la medida que crean que el conocimiento es certero e inmodificable, percibirían mayores beneficios de la evaluación docente para su desarrollo profesional.

Por último, según ubicación geográfica, los análisis arrojan la presencia de correlaciones inversas para el contexto urbano y rural, como se muestra en la Tabla 4. 
Tabla 4

Correlaciones entre Dimensiones de las Creencias Epistemológicas y Dimensiones de las Consecuencias Percibidas de la Evaluación Docente, según Ubicación Geográfica

\begin{tabular}{llcc}
\hline Ubicación geográfica & \multicolumn{1}{c}{ Dimensión } & Autorreflexión & Trabajo colaborativo \\
\cline { 3 - 4 } Rural & Estructura del conocimiento & $-0,309$ & $-0,040$ \\
& & $(<0,001)$ & $(0,628)$ \\
& Estabilidad del conocimiento & $-0,128$ & $-0,190$ \\
& & $(0,118)$ & $(0,020)$ \\
& Control del aprendizaje & 0,074 & 0,072 \\
& & $0,370)$ & $(0,380)$ \\
& Velocidad del aprendizaje & 0,080 & 0,033 \\
& & $(0,333)$ & $(0,693)$ \\
& Estructura del conocimiento & 0,058 & $-0,056$ \\
& & 0,559 & $(0,580)$ \\
& Estabilidad del conocimiento & $-0,165$ & $-0,318$ \\
& & $(0,098)$ & $(0,001)$ \\
& Control del aprendizaje & $-0,047$ & $(0,035$ \\
& & $(0,638)$ & $-0,060$ \\
& Velocidad del aprendizaje & 0,129 & $(0,551)$ \\
\hline
\end{tabular}

Nota. Entre paréntesis aparece $p$.

Los resultados evidencian una relación inversa débil entre las dimensiones Estructura del Conocimiento y Autorreflexión en el contexto rural. Lo anterior implica que a medida que los docentes de contextos rurales manifiestan menor acuerdo con la creencia de que el conocimiento es un todo integrado aumenta la percepción de que la evaluación permite mejorar, a partir de la reflexión sobre las propias prácticas.

Por otro lado, se encuentran relaciones inversas débiles entre las dimensiones Estabilidad del Conocimiento y Trabajo Colaborativo en contextos urbanos. Los datos muestran que los docentes de contextos urbanos que están de acuerdo con las creencias respecto de que el conocimiento es inmodificable y certero, declaran una mayor percepción de que la evaluación docente permite aprender de la práctica, a través del trabajo colaborativo con pares.

No se encontraron relaciones entre las dimensiones Control del Aprendizaje y Velocidad del Aprendizaje con Autorreflexión y Trabajo Colaborativo, según variables sociodemográficas y ubicación geográfica.

Finalmente, el modelo de ecuaciones estructurales integrado arrojó resultados consistentes con los análisis anteriores, mostrando los efectos previamente reportados respecto de la relación entre estructura y estabilidad del conocimiento y la autorreflexión y el trabajo colaborativo (Ver Figura 1), aun cuando se controla por resultados en la evaluación docente, edad, género, años de experiencia, estudios de diplomado o certificaciones, estudios de postgrado y, finalmente, por el anidamiento en colegios. Se evaluó un modelo incorporando también Control del aprendizaje y Velocidad del aprendizaje, pero de forma consistente con los análisis previos no mostraron efectos significativos y por ende no es incluido en estos resultados.

El ajuste del modelo fue bueno, con un valor de 0,018 en la raíz del error cuadrático medio de aproximación (RMSEA), un valor de 0,978 en el índice comparativo de Bentler-Bonett (CFI) y un valor de 0,073 en la raíz cuadrada del promedio de residuales ajustados estandarizada (SRMR). En el caso de RMSEA, los valores menores a 0,05 indican un buen ajuste y mayores a 0,08 indican un mal ajuste. Para el índice CFI, valores superiores a 0,95 indican un buen ajuste del modelo. Los valores de referencia máximos a partir de los cuales se considera que el SRMR indica buen ajuste varían de menos a más exigencia entre 0,08 y a 0,05 (Hu y Bentler, 1999; Schermelleh-Engel et al., 2003). Sin embargo, la prueba $x^{2}$ fue estadísticamente significativa usando un alfa de 0,05 $\left(\chi^{2}(1407)=1524,835, p=0,015\right)$.

La Figura 1 resume los resultados del modelo, en donde autorreflexión y trabajo colaborativo son predichos en regresiones múltiples por los atributos de estructura y estabilidad del conocimiento y por todos los factores de control presentados al lado derecho del diagrama. Los atributos de estructura y estabilidad del 
conocimiento, como los de autorreflexión y trabajo colaborativo, fueron modelados usando análisis factoriales confirmatorios generalizados, mientras que los atributos restantes fueron modelados de forma observada.

\section{Figura 1}

\section{Modelo de Ecuaciones Estructurales}

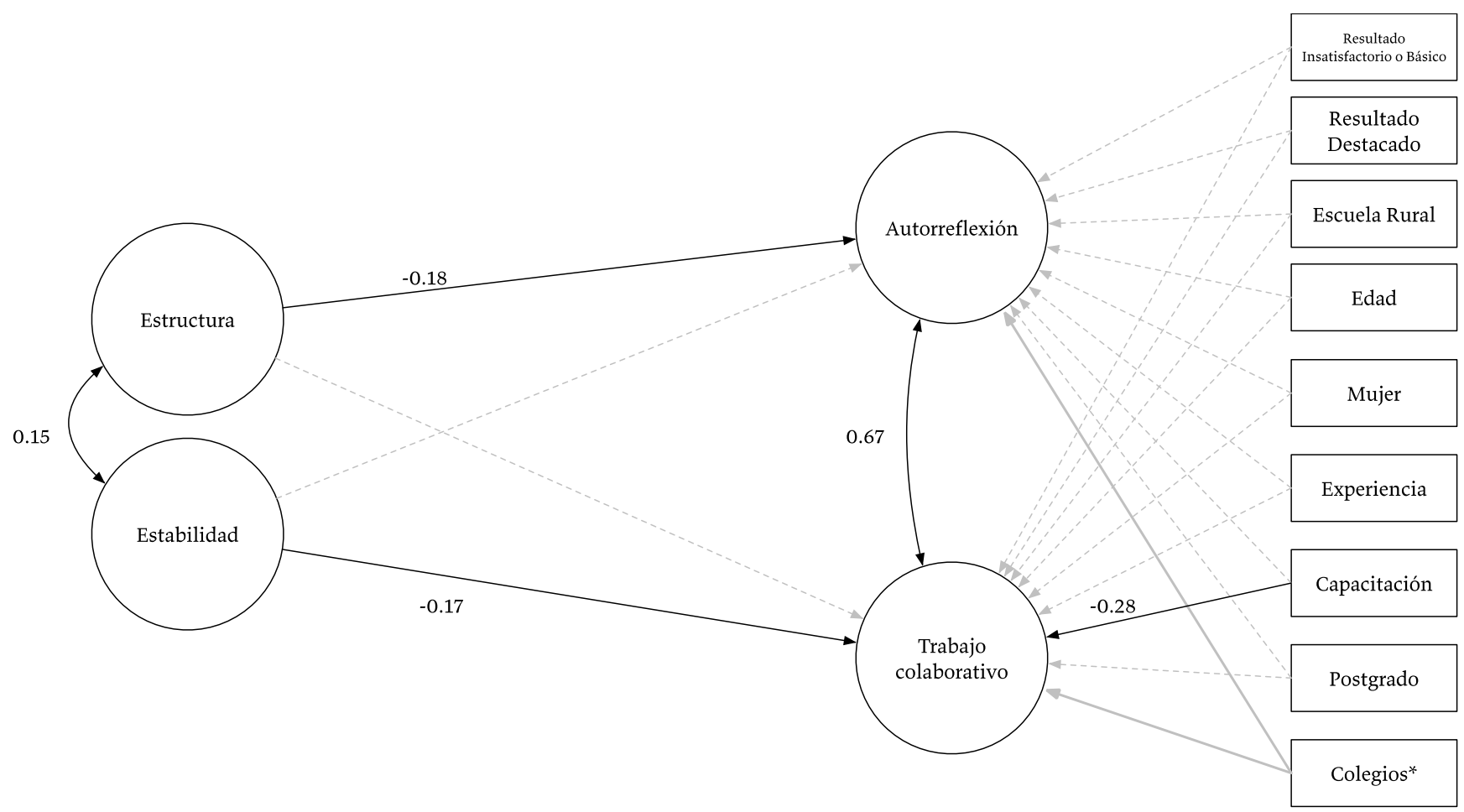

Nota. Los atributos latentes de estructura y estabilidad del conocimiento y los de autorreflexión y trabajo colaborativo están representados con círculos. Los factores considerados como controles observados en la regresión múltiple están expresados con rectángulos a la derecha. La covariable colegios representa los efectos fijos asociados a cada colegio, usados para controlar el anidamiento de este factor. Las flechas continuas negras muestran los efectos significativos y los coeficientes reportados están estandarizados.

Los únicos efectos que fueron encontrados al evaluar todos los factores en su conjunto fueron estructura del conocimiento sobre autorreflexión, en donde un aumento en una unidad de estructura del conocimiento predice negativamente - 0,18 desviaciones estándar de autorreflexión ( $\mathrm{z}=-4,491, p<0,001)$ y estabilidad del conocimiento sobre trabajo colaborativo, en donde un aumento en una unidad de estabilidad del conocimiento predice negativamente $-0,17$ desviaciones estándar de trabajo colaborativo $(\mathrm{z}=-4,049, p<0,001)$. La única variable de control que mostró algún efecto fue la variable indicadora que marcaba el contar con estudios de postítulo o diplomado, lo cual también predecía inversamente el trabajo colaborativo $(\mathrm{z}=-1.996, p=0,46)$.

\section{Discusión}

El desarrollo profesional es fundamental para la calidad de la educación y los sistemas de evaluación docente pueden contribuir a su fortalecimiento (Isoré, 2009; Stronge, 2006). Sin embargo, no todos los estudios concuerdan en el impacto positivo de estos en el desarrollo profesional docente (Isoré, 2009), dadas algunas deficiencias de los sistemas de evaluación (Tucker, 1997), como su desacoplamiento con la realidad y complejidad de la profesión docente (Su et al., 2017), y otros factores contextuales, como la cultura o clima escolar o la percepción de líderes escolares respecto de la evaluación docente (Taut et al., 2011). Incluso estudios enfocados en factores individuales han encontrado que tener creencias favorables respecto de la retroalimentación y trabajo colaborativo que inducen los sistemas de evaluación no tiene impacto en la motivación para el cambio a partir de la evaluación docente (Reddy et al., 2018). 
El presente estudio confirma que no siempre se encuentra un impacto positivo de la evaluación docente en el desarrollo profesional. Además, sus resultados se suman a la escasa evidencia existente de que factores individuales como las creencias epistemológicas, pueden ser un elemento importante para dilucidar esta relación, considerando también la influencia de variables contextuales como lo urbano y rural. Lo anterior da cuenta de que las políticas educativas que establecen estándares uniformes y a nivel país no tienen el mismo impacto en todas las personas y contextos a los cuales van dirigidas.

Los hallazgos de este estudio dan cuenta de que aquellos profesores que mantenían creencias menos sofisticadas, aludiendo a que el conocimiento está conformado por piezas desordenadas y que además es inmodificable y certero, tenían una mayor percepción de que la evaluación docente favorece el desarrollo profesional, a través de la autorreflexión sobre la práctica y el trabajo colaborativo con pares. Por el contrario, aquellos docentes que mantenían creencias sofisticadas declaraban un menor efecto y uso de la evaluación docente para su desarrollo profesional.

Tal hallazgo difiere de autores que señalan que el aprendizaje a nivel profesional análogamente está relacionado con el grado de elaboración de las creencias epistemológicas (Guerra Zamora y Sebastián Balmaceda (2015) y que el pensamiento de alto nivel se asocia con operar con creencias epistemológicas más sofisticadas (Harteis et al, 2006).

Estos resultados llevan a hipotetizar que aquellos docentes con creencias ingenuas perciben la evaluación docente como un instrumento creíble y en el que se puede confiar, valorándolo por sobre otras piezas de evidencia y, quizás incluso, por sobre su propio criterio profesional. Por otra parte, los docentes que creen que el conocimiento es integrado y tentativo, optan por no considerar la evaluación docente como único criterio, sino más bien confiar en su experiencia y práctica para la mejora de la enseñanza, haciendo menor uso de la evaluación docente.

Una posible explicación a este hallazgo, que difiere de los resultados encontrados en otras investigaciones que buscaban conocer la relación entre las creencias epistemológicas y el desarrollo profesional, radica en que la presente investigación estuvo focalizada en un desarrollo profesional en particular, es decir, en el que tiene lugar a partir de la evaluación docente chilena. Esto haría suponer que las características del propio Sistema de Evaluación del Desempeño Docente Nacional estarían limitando el desarrollo profesional en aquellos docentes con creencias epistemológicas sofisticadas. Para ellos, la evaluación docente podría tratarse de un instrumento "básico" para su aprendizaje o progreso profesional, considerando que, como señalan algunos autores, los individuos que creen en la complejidad del conocimiento tienden a reconocer esta complejidad en los temas a discutir, así como a tomar múltiples perspectivas de análisis (Schommer-Aikins \& Hutter, 2002). Este hallazgo confirma lo reportado por otros estudios que revelan la importancia de que los sistemas de evaluación reconozcan las necesidades, creencias o experiencias de los docentes, para que efectivamente tengan un impacto en el desarrollo docente y, finalmente, en el logro estudiantil (Reddy et al, 2018).

Variables sociodemográficas, como los resultados de la evaluación, los años de experiencia y el grado educativo, no fueron significativos en la relación entre creencias epistemológicas y desarrollo profesional a partir de la evaluación docente, según el modelo final aplicado. Investigaciones previas tampoco reportan hallazgos significativos en esta relación respecto del grado educacional, pero sí reportan correlaciones inversas entre años de experiencia y motivación de los docentes por cambiar (Reddy et al., 2018) y entre la satisfacción y la evaluación y los beneficios obtenidos de ella con la motivación hacia el cambio (Delvaux et al., 2013; Reddy et al., 2018). La ausencia de impacto de los resultados de la evaluación en el desarrollo profesional encontrada en este estudio invita a potenciar la evaluación docente como un sistema que realmente tenga un impacto positivo y concreto en la vida profesional de los docentes, para así impulsar una motivación hacia el desarrollo profesional.

Respecto de las diferencias en las relaciones entre creencias epistemológicas y consecuencias percibidas de la evaluación docente según ubicación geográfica, los resultados dan cuenta de que en el contexto rural los docentes que mantenían creencias ingenuas sobre el conocimiento manifestaron mayor autorreflexión sobre la práctica a partir de la evaluación. Por otra parte, los resultados indican que quienes se desempeñaban en contextos urbanos, en la medida que creían que el conocimiento es inmodificable y certero tendían a usar más el trabajo colaborativo. Estos resultados evidencian que aquellos docentes que no cuentan con herramientas internas recurren a herramientas externas para hacer frente a los desafíos de su práctica, lo que es mucho más efectivo en contextos urbanos, en donde se da con mayor probabilidad el trabajo colaborativo. Los contextos rurales se encuentran en una situación menos favorable para compartir experiencias y reflexionar con otros sobre el desempeño. Castillo-Miranda et al. (2017) señalan que el proceso de evaluación docente en 
contextos rurales es más solitario, contando con opciones mínimas o nulas de preguntar a otros colegas durante el proceso evaluativo.

En conclusión, de acuerdo con la OECD (2015), aunque la definición de estándares de calidad profesionales y la evaluación de la correspondencia de los docentes con estos estándares puede contribuir a la profesionalización docente y al fortalecimiento de la carrera docente chilena, los hallazgos de este estudio entregan información relevante para las políticas educativas y los posibles esfuerzos de mejora del sistema de evaluación docente nacional para acercarse efectivamente al objetivo principal, es decir, fortalecer las competencias docentes.

Por un lado, los resultados advierten que el proceso de evaluación docente no tiene el mismo impacto en todos los docentes de acuerdo a visiones propias sobre el conocimiento. Visibilizar estas diferencias permitiría reformar el sistema de evaluación, considerando las necesidades docentes y orientar el sistema no solo hacia abordar las debilidades, sino también a potenciar aquellas competencias que ya están desarrolladas. Aunque el fortalecimiento del profesorado pudiese atribuirse a la responsabilidad de cada docente respecto de la mejora de sus competencias, es el sistema educativo, como señala Roa-Tampe (2017), el que debe potenciar y guiar el desarrollo profesional docente para mejorar la enseñanza.

Asimismo, estos hallazgos invitan a considerar que, dada la diversidad docente y sus contextos de desempeño, un sistema único, generalizado y estandarizado, no puede abordar la heterogeneidad y la complejidad de la labor docente. Por lo tanto, no se puede suponer que un único sistema puede abordar necesidades de contextos tan distintos como el urbano y rural, siendo importante orientar un sistema de evaluación docente contextualizado, que permita abordar desafíos únicos de determinados entornos sociales y así orientar el desarrollo profesional de acuerdo a sus ambientes de desempeño. Lo anterior revela como estrategia la importancia de potenciar y posicionar los microcentros rurales como un espacio de reflexión pedagógica y colaboración docente (MINEDUC, 2016), considerando que los profesores, en promedio en la OECD, reportan que una de las maneras de formación continua que ha tenido un mayor impacto en ellos son aquellas basadas en la colaboración y los enfoques de colaboración para la docencia (OECD, 2019).

Evidentemente, el uso y efecto formativo de la evaluación docente depende de múltiples factores; sin embargo, considerar elementos individuales en este fenómeno permite reconocer la importancia de la evaluación docente para la formación continua a través de la autorreflexión y el trabajo colaborativo en aquellos docentes que manifiestan mayores necesidades. Asimismo, permite reconocer que para otros docentes el sistema de evaluación nacional se estaría alejando de su propósito formativo, siendo imprescindible para evitar aquello revisar y replantear su metodología.

Entre las limitaciones del estudio cabe señalar que, si bien el instrumento construido obtuvo índices de Cronbach y de validez altos, no se llevó a cabo el procedimiento de validación completo, lo que pudo afectar los resultados obtenidos. Por otra parte, la naturaleza autorreportada de la información y la muestra autoseleccionada puede haber afectado la calidad de los datos obtenidos. Otra limitante alude al estudio de un fenómeno multifactorial a través de la relación entre variables a nivel individual, lo que se intentó contrarrestar considerando un factor de contexto, la ubicación geográfica, la cual probablemente influye en el tipo de formación o desarrollo profesional que persigue un profesor a partir de la evaluación docente. Sin embargo, para futuras investigaciones sería útil considerar otros factores relevantes a nivel organizacional y de cultura docente, como el liderazgo directivo. Asimismo, un enfoque cualitativo con estudios de casos también podría ser interesante para obtener información sobre los procesos subyacentes en los docentes que llevan a hacer uso de la evaluación docente para su desarrollo profesional, todo con miras a alcanzar una panorámica más completa de cómo la evaluación docente podría contribuir de mejor manera al desarrollo profesional y así cumplir a cabalidad con su propósito formativo.

\section{Referencias}

Accomplished California Teachers. (2015). A coherent system of teacher evaluation for quality teaching. Education Policy Analysis Archives, 23, Artículo 17. https://doi.org/10.14507/epaa.v23.2006

Aprueba reglamento sobre evaluación docente, Decreto 192, Ministerio de Educación de Chile (2004). https://www.docentemas.cl/descargas/marco_legal/ReglamentoEvaluacionDocente.pdf

Araya, C. (2005). Informe para estudio de validación AEP 2005 [Manuscrito no publicado]. Centro de Medición MIDE UC, Pontificia Universidad Católica de Chile.

Avalos, B. \& Assael, J. (2006). Moving from resistance to agreement: The case of the Chilean teacher performance evaluation. International Journal of Educational Research, 45(4-5), 254-266. https://doi.org/10.1016/j.ijer.2007.02.004 
Bandura, A. (2008). Reconstrual of "free will" from the agentic perspective of social cognitive theory. En J. Baer, J. C. Kaufman \& R. F. Baumeister (Eds.), Are we free? Psychology and free will (pp. 86-127). Oxford University Press. https://doi.org/10.1093/acprof:oso/9780195189636.003.0006

Bendixen, L. D., Schraw, G. \& Dunkle, M. E. (1998). Epistemic beliefs and moral reasoning. The Journal of Psychology, 132(2), 187-200. https://doi.org/10.1080/00223989809599158

Biesta, G., Priestley, M. \& Robinson, S. (2015). The role of beliefs in teacher agency. Teachers and Teaching, 21(6), 624-640. https://doi.org/10.1080/13540602.2015.1044325

Bonifaz, R. (2011). Origen de la evaluación docente y su conexión con las políticas públicas en educación. En J. Manzi, R. González \& Y. Sun (Eds.), La evaluación docente en Chile (pp. 13-32). Pontificia Universidad Católica de Chile.

Cabezas, V., Hochschild, H. \& Medeiros, M. P. (2019). Los desafíos pendientes en la ejecución de la nueva política docente: ¿es suficiente con la ley? En A. Carrasco \& L. M. Flores (Eds.), De la reforma a la transformación: capacidades, innovaciones y regulación de la educación chilena (pp. 29-67). Ediciones UC.

Castillo-Miranda, S. R., Williamson Castro, G. \& Hidalgo-Standen, C. (2017). La evaluación del desempeño docente desde la perspectiva de profesores de educación rural. Educación y Educadores, 20(3), 364-381. https://doi.org/10.5294/edu.2017.20.3.2

Castro, P. J., Krause, M. \& Frisancho, S. (2015). Teoría del cambio subjetivo: aportes desde un estudio cualitativo con profesores. Revista Colombiana de Psicología, 24(2), 362-379. https://doi.org/10.15446/rcp.v24n2.44453

Centro de Estudios MINEDUC. (2015). Docentes en Chile: resultados de la encuesta TALIS 2013. Gobierno de Chile, Ministerio de Educación.https://centroestudios.mineduc.cl/wp-content/uploads/sites/100/2017/06/evidencia-final_marzo_2015.pdf

Centro de Estudios MINEDUC. (2018). Estadísticas de la educación 2017 [Conjunto de datos]. Gobierno de Chile, Ministerio de Educación. https://centroestudios.mineduc.cl/wp-content/uploads/sites/100/2018/12/ANUARIO-MINEDUC VERSION-BAJA.pdf

Centro de Perfeccionamiento, Experimentación e Investigaciones Pedagógicas. (Diciembre de 2008). Marco para la buena enseñanza (7 ${ }^{\mathrm{a}}$ ed.). Gobierno de Chile, Ministerio de Educación. https://www.docentemas.cl/docs/MBE2008.pdf

Centro de Perfeccionamiento, Experimentación e Investigaciones Pedagógicas. (Agosto de 2019). Resultados nacionales evaluación docente 2018 [Diapositivas de PowerPoint]. Gobierno de Chile, Ministerio de Educación. https://www.cpeip.cl/wpcontent/uploads/2019/08/Resultados-Evaluaci\%C3\%B3n-Docente-2018.pdf

Chan, K. -w. \& Elliott, R. G. (2002). Exploratory study of Hong Kong teacher education students' epistemological beliefs: Cultural perspectives and implications on beliefs research. Contemporary Educational Psychology, 27(3), 392-414. https://doi.org/10.1006/ceps.2001.1102

Cox, C. (2007). Educación en el bicentenario: dos agendas y calidad de la política. Pensamiento Educativo, 40(1), 175-204. http://pensamientoeducativo.uc.cl/index.php/pel/article/download/404/822

de Vries, S., van de Grift, W. J. C. M. \& Jansen, E. P. W. A. (2014). How teachers' beliefs about learning and teaching relate to their continuing professional development. Teachers and Teaching, 20(3), 338-357. https://doi.org/10.1080/13540602.2013.848521

Delvaux, E., Vanhoof. J., Tuytens, M., Vekeman, E., Devos, G. \& Van Petegem, P. (2013). How may teacher evaluation have an impact on professional development? A multilevel analysis. Teaching and Teacher Education, 36, 1-11. https://doi.org/10.1016/j.tate.2013.06.011

Desimone, L. (2009). Improving impact studies of teachers' professional development: Toward better conceptualizations and measures. Educational Researcher, 38(3), 181-199. https://doi.org/10.3102/0013189X08331140

DocenteMás. (s.f.a). Qué es la evaluación docente. Gobierno de Chile, Ministerio de Educación. https://www.docentemas.cl/pages/quienessomos/que-es-la-evaluacion-docente

DocenteMás (s.f.b). Resultados y consecuencias: los niveles de desempeño. Gobierno de Chile, Ministerio de Educación. https://www.docentemas.cl/pages/conozca-la-evaluacion/niveles-de-desempeno

Eurydice. (2008). Levels of autonomy and responsibilities of teachers in Europe. https://doi.org/10.2766/35479

Fang, Z. (1996). A review of research on teacher beliefs and practices. Educational Research, 38(1), 47-65. https://doi.org/10.1080/0013188960380104

Feiman-Nemser, S. (2001). From preparation to practice: Designing a continuum to strengthen and sustain teaching. Teachers College Record, 103(6), 1013-1055. https://doi.org/10.1111/0161-4681.00141

Flores, M. A. (2012). The implementation of a new policy on teacher appraisal in Portugal: How do teachers experience it at school? Educational Assessment, Evaluation and Accountability, 24(4), 351-368. https://doi.org/10.1007/s11092-012-9153-7

Flotts, M. P. \& Abarzúa, A. (2011). El modelo de evaluación y los instrumentos. En J. Manzi, R. González \& Y. Sun (Eds.), La evaluación docente en Chile (pp. 37-61). Pontificia Universidad Católica de Chile.

Fullan, M. \& Stiegelbauer, S. M. (1997). El cambio educativo: guía de planeación para maestros. Trillas.

García, M. R. \& Sebastián, C. (2011). Creencias epistemológicas de estudiantes de pedagogía en educación parvularia, básica y media: ¿diferencias en la formación inicial docente? Psykhe, 20(1), 29-43. https://doi.org/10.4067/S0718-22282011000100003

Geijsel, F. P., Sleegers, P. J. C., Stoel, R. D. \& Krüger, M. L. (2009). The effect of teacher psychological and school organizational and leadership factors on teachers' professional learning in Dutch schools. The Elementary School Journal, 109(4), 406-427. https://doi.org/10.1086/593940

Gore, J., Lloyd, A., Smith, M., Bowe, J., Ellis, H. \& Lubans, D. (2017). Effects of professional development on the quality of teaching: Results from a randomised controlled trial of quality teaching rounds. Teaching and Teacher Education, 68, 99-113. https://doi.org/10.1016/j.tate.2017.08.007

Guerra Zamora, P. \& Sebastián Balmaceda, C. (2015). Creencias epistemológicas en profesores que postulan al Programa de Acreditación de Excelencia Pedagógica: análisis descriptivos y comparativos entre profesores que se desempeñan en los diferentes niveles de enseñanza. Estudios Pedagógicos, 41(2), 107-125. https://doi.org/10.4067/S0718-07052015000200007

Harteis, C., Gruber, H. \& Lehner, F. (2006). Epistemological beliefs and their impact on work, subjectivity and learning. En S. Billett, T. Fenwick \& M. Somerville (Eds.), Work, subjectivity and learning: Understanding learning through working life (pp. 123-140). Springer Netherlands. https://doi.org/10.1007/1-4020-5360-6_8

Heneman III, H. G. \& Milanowski, A. T. (2003). Continuing assessment of teacher reaction to a standards-based teacher evaluation system. Journal of Personnel Evaluation in Education, 17(2), 173-195. https://doi.org/10.1023/B:PEEV.0000032427.99952.02

Hofer, B. K. (2004). Exploring the dimensions of personal epistemology in differing classroom contexts: Student interpretations during the first year of college. Contemporary Educational Psychology, 29(2), 129-163. https://doi.org/10.1016/ i.cedpsych.2004.01.002

Hofer, B. K. \& Pintrich, P. R. (1997). The development of epistemological theories: Beliefs about knowledge and knowing and their relation to learning. Review of Educational Research, 67(1), 88-140. https://doi.org/10.3102/00346543067001088

$\mathrm{Hu}$, L. \& Bentler P. M. (1999). Cutoff criteria for fit indexes in covariance structure analysis: Conventional criteria versus new alternatives. Structural Equation Modeling, 6(1), 1-55. https://doi.org/10.1080/10705519909540118 
Hyman, J. (2018). A study of teachers' perceptions of the Texas Teacher Evaluation and Support System [Tesis de doctorado no publicada]. Dallas Baptist University. https:/www.proquest.com/openview/7c53abbf982e6f030a882816f2a9a251/1?pq-origsite=gscholar\&cbl=18750\&diss=y

Isoré, M. (2009). Teacher evaluation: Current practices in OECD countries and a literature review (OECD Education Working Papers $\mathrm{N}^{\circ}$ 23). OECD Publishing. https://doi.org/10.1787/223283631428

Kyriakides, L., Christoforidou, M., Panayiotou, A. \& Creemers, B. P. M. (2017). The impact of a three-year teacher professional development course on quality of teaching: Strengths and limitations of the dynamic approach. European Journal of Teacher Education, 40(4), 465-486. https://doi.org/10.1080/02619768.2017.1349093

Lieberman, A. (1995). Restructuring schools: The dynamics of changing practice, structure, and culture. En A. Lieberman (Ed.), The work of restructuring schools: Building from the ground up (pp. 1-17). Teachers College Press.

Little, J. W. (1993). Teachers' professional development in a climate of educational reform. Educational Evaluation and Policy Analysis, 15(2), 129-151. https://doi.org/10.3102/01623737015002129

Looney, J. (2011). Developing high-quality teachers: Teacher evaluation for improvement. European Journal of Education, 46(4), 440455. https://doi.org/10.1111/j.1465-3435.2011.01492.x

Macfarlane, B. \& Gourlay, L. (2009). The reflection game: Enacting the penitent self. Teaching in Higher Education, 14(4), 455-459. https://doi.org/10.1080/13562510903050244

Ministerio de Educación. (2016). Sistema de desarrollo profesional docente: una mejora trascendental para los profesores. REVEDUC: Revista de Educación, 374, 6-13. https://bibliotecadigital.mineduc.cl/bitstream/handle/20.500.12365/5050/reveduc\%20374.pdf?.sequence=1\&isAllowed=y

Ministerio de Educación. (s.f.). Desarrollo profesional en territorios rurales: la escuela, los docentes y las redes profesionales. Gobierno de Chile. https://rural.mineduc.cl/desarrollo-profesional-territorios-rurales/

Nussbaum, E. M. \& Bendixen, L. D. (2003). Approaching and avoiding arguments: The role of epistemological beliefs, need for cognition, and extraverted personality traits. Contemporary Educational Psychology, 28(4), 573-595. https://doi.org/10.1016/S0361-476X(02)00062-0

Opfer, V. D., Pedder, D. G. \& Lavicza, Z. (2011). The role of teachers' orientation to learning in professional development and change: A national study of teachers in England. Teaching and Teacher Education, 27(2), 443-453. https://doi.org/ 10.1016/j.tate.2010.09.014

Organisation for Economic Co-operation and Development. (20 de marzo de 2015). Reformulando la carrera docente en Chile: evidencia internacional seleccionada. https://www.oecd.org/education/school/Reformulando-la-carrera-docente-en-chile.pdf

Organisation for Economic Co-operation and Development. (2019). TALIS 2018 results (volume I): Teachers and school leaders as lifelong learners. OECD Publishing. https://doi.org/10.1787/1d0bc92a-en

Paechter, M. R., Rebmann, K. Schloemer, T., Mokwinski, B., Hanekamp, Y. \& Arendasy, M. (2013). Development of the Oldenburg Epistemic Beliefs Questionnaire (OLEQ), a German questionnaire based on the Epistemic Belief Inventory (EBI). Current Issues in Education, 16(1), Artículo 2. https://cie.asu.edu/ojs/index.php/cieatasu/article/view/1035

Peterson, K. D. \& Peterson, C. A. (2006). Effective teacher evaluation: A guide for principals. Corwin Press. https://doi.org/10.4135/9781412990219

Putnam, R. T. \& Borko, H. (2000). What do new views of knowledge and thinking have to say about research on teacher learning? Educational Researcher, 29(1), 4-15. https://doi.org/10.3102/0013189X029001004

Reddy, L. A., Dudek, C. M., Peters, S., Alperin, A., Kettler, R. J. \& Kurz, A. (2018). Teachers' and school administrators' attitudes and beliefs of teacher evaluation: A preliminary investigation of high poverty school districts. Educational Assessment, Evaluation and Accountability, 30(1), 47-70 https://doi.org/10.1007/s11092-017-9263-3

Reddy, L. A., Kettler, R. J. \& Kurz, A. (2015). School-wide educator evaluation for improving school capacity and student achievement in high-poverty schools: Year 1 of the School System Improvement Project. Journal of Educational and Psychological Consultation, 25(2-3), 90-108. https://doi.org/10.1080/10474412.2014.929961

Richardson, V. (1996). The role of attitudes and beliefs in learning to teach. En J. Sikula (Ed.), Handbook of research on teacher education. Second edition (pp. 102-119). Macmillan.

Richter, D., Kunter, M., Klusmann, U., Lüdtke, O. \& Baumert, J. (2011). Professional development across the teaching career: Teachers' uptake of formal and informal learning opportunities. Teaching and Teacher Education, 27(1), 116-126. https://doi.org/10.1016/j.tate.2010.07.008

Roa-Tampe, K. A. (2017). La evaluación docente bajo la óptica del desarrollo profesional: el caso chileno. Educación y Educadores, 20(1), 41-61. https://doi.org/10.5294/edu.2017.20.1.3

Rubio González, J., Andrade Daigre, P., Fravega Araneda, G., Macalusso Salgado, S. \& Soto Sandoval, A. (2019). Factores psico-socioambientales asociados al estrés laboral en profesores chilenos del ámbito rural y urbano. Propósitos y Representaciones, 7(3), 300322. https://doi.org/10.20511/pyr2019.v7n3.358

Runhaar, P., Sanders, K. \& Yang, H. (2010). Stimulating teachers' reflection and feedback asking: An interplay of self-efficacy, learning goal orientation, and transformational leadership. Teaching and Teacher Education, 26(5), 1154-1161. https://doi.org/10.1016/j.tate.2010.02.011

Santiago, P. \& Benavides, F. (1-2 de diciembre de 2009). Teacher evaluation: A conceptual framework and examples of country practices [Exposición]. OECD Workshop Towards a Teacher Evaluation Framework in Mexico: International Practices, Criteria and Mechanisms, Ciudad de México, México. http://www.oecd.org/edu/school/44568106.pdf

Schermelleh-Engel, K., Moosbrugger, H. \& Müller, H. (2003). Evaluating the fit of structural equation models: Tests of significance and descriptive goodness-of-fit measures. Methods of Psychological Research, 8(2), 23-74. https://www.researchgate.net/publication/251060246_Evaluating_the_Fit_of_Structural_Equation_Models_Tests_of_Significance_ and_Descriptive_Goodness-of-Fit_Measures

Schommer, M. (1990). Effects of beliefs about the nature of knowledge on comprehension. Journal of Educational Psychology, 82(3), 498504. https://doi.org/10.1037/0022-0663.82.3.498

Schommer-Aikins, M. \& Hutter, R. (2002). Epistemological beliefs and thinking about everyday controversial issues. The Journal of Psychology, 136(1), 5-20. https://doi.org/10.1080/00223980209604134

Schraw, G., Bendixen, L. D. \& Dunkle, M. E. (2002). Development and validation of the Epistemic Belief Inventory (EBI). En B. K. Hofer \& P. R. Pintrich (Eds.), Personal epistemology: The psychology of beliefs about knowledge and knowing (pp. 261-275). Lawrence Erlbaum.

Smylie, M. A. (2014). Teacher evaluation and the problem of professional development. Mid-Western Educational Researcher, 26(2), 97111. https://www.mwera.org/MWER/volumes/v26/issue2/v26n2-Smylie-POLICY-BRIEFS.pdf

Soebari, T. \& Aldridge J. (2016). Investigating the differential effectiveness of a teacher professional development programme for rural and urban classrooms in Indonesia. Teacher Development, 20(5), 701-722. https://doi.org/10.1080/13664530.2016.1185031

Stronge, J. H. (1995). Balancing individual and institutional goals in educational personnel evaluation: A conceptual framework. Studies in Educational Evaluation, 21(2), 131-151. https://doi.org/10.1016/0191-491X(95)00010-R 
Stronge, J. H. (Ed.). (2006). Evaluating teaching: A guide to current thinking and best practice. Second edition. Corwin Press. https://doi.org/10.4135/9781412990202

$\mathrm{Su}, \mathrm{Y} .$, Feng, L. \& Hsu, C. -H. (2017). Accountability or authenticity? The alignment of professional development and teacher evaluation. Teachers and Teaching, 23(6), 717-728. https://doi.org/10.1080/13540602.2016.1255189

Taut, S., Santelices, V., Araya, C. \& Manzi, J. (2010). Theory underlying a national teacher evaluation program. Evaluation and Program Planning, 33(4), 477-486. https://doi.org/10.1016/j.evalprogplan.2010.01.002

Taut, S., Santelices, M. V., Araya, C. \& Manzi, J. (2011). Perceived effects and uses of the national teacher evaluation system in Chilean elementary schools. Studies in Educational Evaluation, 37(4), 218-229. https://doi.org/10.1016/j.stueduc.2011.08.002

Taut, S., Santelices, M. V. \& Stecher, B. (2012). Validation of a national teacher assessment and improvement system. Educational Assessment, 17(4), 163-199. https://doi.org/10.1080/10627197.2012.735913

Tucker, P. D. (1997). Lake Wobegon: Where all teachers are competent (or, have we come to terms with the problem of incompetent teachers?). Journal of Personnel Evaluation in Education, 11(2), 103-126. https://doi.org/10.1023/A:1007962302463

Tuytens, M. \& Devos, G. (2011). Stimulating professional learning through teacher evaluation: An impossible task for the school leader? Teaching and Teacher Education, 27(5), 891-899. https://doi.org/10.1016/j.tate.2011.02.004

Fecha de recepción: Agosto de 2019.

Fecha de aceptación: Septiembre de 2020. 